\title{
Intermittent Diarrhea as a Delayed Presentation of Percutaneous Endoscopic Gastrostomy (PEG)-Associated Fistula
}

\author{
Ghan-Shyam Lohiya, MS, MD, Lilia Tan-Figueroa, MD, and Vamsi Krishna, MD
}

A 60-year-old patient with severe dysphagia, weight loss, and recurrent aspiration pneumonia required a percutaneous endoscopic gastrostomy (PEG) for long-term feeding. After 24 uneventful days, she developed an enigmatic recurring but intermittent diarrhea. On day 62, staff noted a feculent odor from her gastrostoma, along with undigested formula in her stools. This prompted her hospitalization. A plain abdominal radiograph demonstrated the PEG tube in the upper abdomen, but could not differentiate if its tip was misplaced. Next, an abdominal barium-contrast computed tomography scan was performed but was inadvertently misinterpreted as normal. Finally, a colonoscopy demonstrated that the tip of the PEG tube was malpositioned in the transverse colon, resulting in a colocutaneous fistula (CCF). The PEG tube was withdrawn uneventfully through the gastrostoma. A laparotomy was performed. Strong adhesions were found between the stomach and the colon; these were lysed and the CCF tract was excised. The patient recovered. CCF should be considered in the differential diagnosis of PEG patients with unexplained diarrhea even if the diarrhea is delayed or intermittent; the diagnosis should be confirmed by a tubogram. (J Am Board Fam Med 2010;23:681-684.)

Keywords: Colocutaneous Fistulization, Percutaneous Endoscopic Gastrostomy, PEG, Tubogram, Gastroinestinal Disorders, Developmental Disabilities, Medical Errors, Case Reports

Percutaneous endoscopic gastrostomy (PEG) is the preferred mode of providing enteral access for patients who require long-term, nonoral nutritional support. In the United States more than 216,000 PEGs are performed annually. ${ }^{1}$ PEG is generally a safe procedure, but may be complicated by tube dislodgment, peristomal leakage, local infection, gastric perforation, bleeding, aspiration, ileus, pneumoperitnoeum, bowel trauma, and, rarely, a

This article was externally peer reviewed.

Submitted 30 November 2009; revised 8 April 2010; accepted 20 April 2010.

From the Fairview Developmental Center, Costa Mesa, CA (G-SL, LT-F); and the Department of Internal Medicine, University of California, Los Angeles (VK).

Funding: Funding provided by Fairview Developmental Center, Costa Mesa, CA, and the California Department of Developmental Services.

Prior presentation: This work was presented in part at the 39th Quarterly Grand Rounds at Fairview Developmental Center, Costa Mesa, CA, 19 November 2008.

Conflict of interest: none declared.

Corresponding author: Ghan-Shyam Lohiya, MD, MS, Fairview Developmental Center, 2501 Harbor Blvd, Costa Mesa, CA 92626 (E-mail: glohiya@fdc.dds.ca.gov). colocutaneous fistula (CCF). ${ }^{1-10}$ In CCF, the PEG tip is located in the colon instead of the stomach. The formula entering the colon through a CCF remains undigested as it bypasses the small intestine. Classically, patients with a CCF have osmotic watery diarrhea, which is immediate postprandially, occurs regularly after each PEG feed, and starts soon after PEG insertion. Here we present an unusual case in which a CCF manifested with intermittent diarrhea 24 days after an apparently uncomplicated PEG insertion. Although CCFs have been well documented in the gastroenterology literature, they have received little attention in the family practice field. Family physicians who manage nursing home patients may find this case informative for suspecting and appropriately diagnosing a CCF.

\section{Case Report}

Our patient was a resident of a long-term care facility in California for people with severe developmental disabilities (mental retardation, cerebral 
palsy, autism, and/or epilepsy). Of this facility's 480 residents, 99 (21\%) have feeding gastrostomies, which are overwhelmingly PEGs. Our patient was 60 years old and had profound mental retardation, Down syndrome, and tonic-clonic epilepsy. She had no prior abdominal surgery. She weighed $97 \mathrm{lb}$ (44 kg); her height was 5' $(152 \mathrm{~cm})$ and her body mass index was $20 \mathrm{~kg} / \mathrm{m}^{2}$ (normal, 18 to $25 \mathrm{~kg} / \mathrm{m}^{2}$ ). She had dysphagia and often choked while eating. Despite precautions to prevent aspiration she had lost $11 \mathrm{lb}(5 \mathrm{~kg})$ and required 2 hospitalizations for aspiration pneumonia during the preceding 5 months. Her treatment team recommended a feeding gastrostomy.

On day 1 of this illness, an experienced gastroenterologist inserted a Bard French \#20 PEG tube per standard protocol. On day 8, mild erythema, induration, white-yellow gummy discharge, and foul odor were noted at the gastostroma, along with a fever of $100.5^{\circ} \mathrm{F}\left(38^{\circ} \mathrm{C}\right)$. Cellulitis was diagnosed and treated with trimethoprim-sulfamethoxazole via PEG and topical antibiotics for 7 days; a wound culture was not performed. The cellulitis decreased around day 19 , but mild stomal erythema persisted.

The patient initially tolerated PEG feedings uneventfully at 60 to $80 \mathrm{~mL} /$ hour. However, on day 24 , she developed an intermittent, watery or semisolid and malodorous diarrhea of an indeterminate etiology. She had no history of gluten intolerance or chronic diarrhea so tests for celiac disease were not performed. Her stool was negative for clostrid- ium difficile toxins A and B (tested by enzymelinked immunoabsorbant assay), occult blood, and ova and parasites. Stool culture grew no pathogenic bacteria. Serum chemistry and blood count were normal. Diarrhea improved with substitution of formula with clear electrolyte fluids, but it did not resolve. No benefit accrued after empiric treatment with metronidazole $1500 \mathrm{mg} /$ day on days 26 to 32, and lactobacillus acidophilus probiotic supplements on days 30 to 61 . To ensure adequate hydration, the patient was treated with intravenous fluids from day 34 to 40, but trials of PEG feeding at 30 to 40 $\mathrm{mL} /$ hour still produced diarrhea. Gastric residues remained normal throughout $(<60 \mathrm{~mL})$. The PEG tube was handled with usual care by licensed staff, and was never extruded or replaced.

On day 62, the patient's caregivers noted a feculent odor from her gastrostoma and undigested formula in her stool after a PEG feeding. This dramatic observation instigated her hospitalization for further investigation. A plain abdominal radiograph demonstrated fecal residue and gas throughout the abdomen in an ileus pattern and the PEG tube in the upper abdomen, but it could not differentiate if the PEG tip was misplaced. Next, an abdominal computerized axial tomography (CAT) scan with an oral barium contrast was inadvertently misinterpreted as normal by a board-certified radiologist (on a subsequent review, the PEG tip was clearly seen in the transverse colon; Figure 1). Finally, a diagnostic colonoscopy revealed the tip of

Figure 1. Computerized axial tomography scan of the abdomen with contrast. The gastrostomy tube is seen as a radiopaque linear density lying obliquely in the middle of the anterior abdominal wall (curved arrow), with its tip in the colon (straight arrow) at the mid-kidney level. The kidneys are seen on either side of the vertebra.

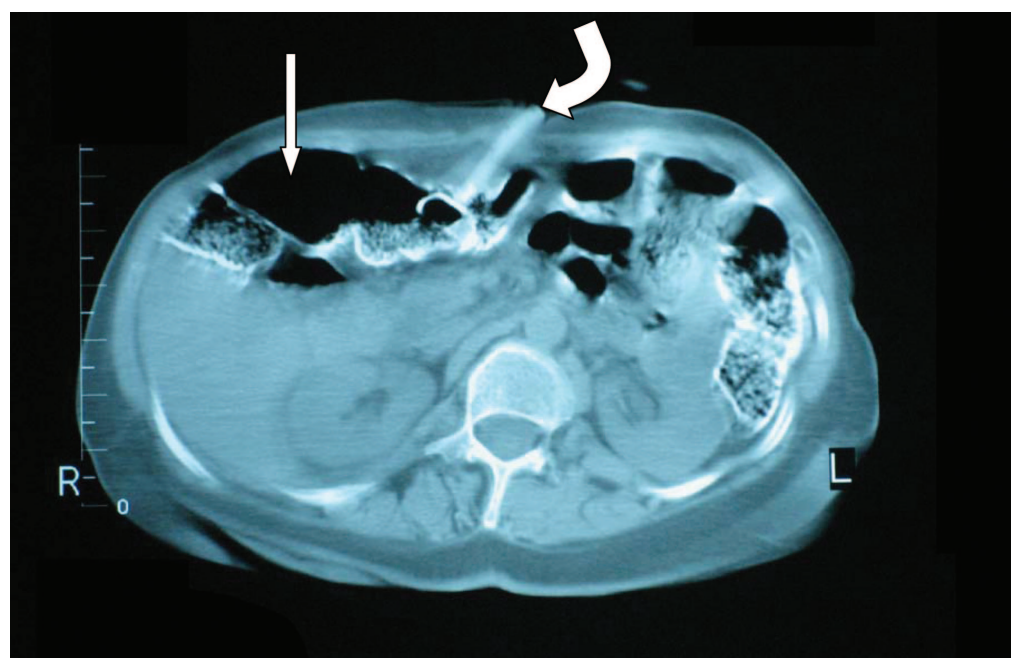


the PEG tube in the transverse colon, which resulted in a CCF (Figure 2). The malpositioned tube was withdrawn through the gastrostoma in the anterior abdominal wall uneventfully. On day 69, a laparotomy was performed to create a reliable surgical gastrostomy. The CCF tract extended directly between the skin and the colon without passage through the stomach; it was excised. Strong adhesions were observed between the stomach and the colon; these were lysed. No gastrocolonic fistula or tract was seen. The patient developed right upper lobe pneumonia postoperatively, which resolved with intravenous antibiotics. She was discharged from the hospital on day 85 .

\section{Discussion}

The most plausible explanation of this CCF is that, during the original PEG insertion, the guide needle passed from the skin into the colon and then entered the stomach. The PEG tube followed that needle retrograde through the stomach, first penetrated the colon, and then exited the skin. This hypothesis is supported by the abdominal computed tomography scan (Figure 1), which shows that the PEG insertion site was too low, at the mid-kidney level near the umbilicus, and thus increased the likelihood of colonic interpositioning.

Figure 2. Colonoscopic demonstration of the tip of the percutaneous endoscopic gastrostomy tube in the transverse colon. The tube's lumen contains fecal matter. The mucosal fold below the tube is a colonic haustration.

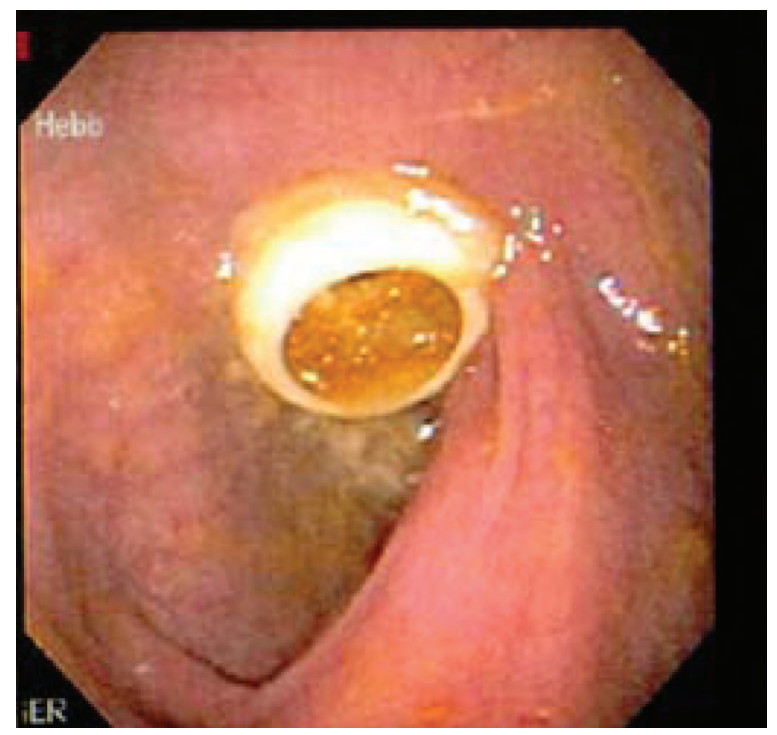

Strong adhesions developed between the stomach and the colon around the PEG tube, creating a gastrocolonic channel. Space between them was virtually eliminated because of PEG-related close apposition and contraction of the fibrous adhesions. This is possibly why no gastrocolonic fistular tract was seen during the laparotomy.

The PEG functioned normally for the first 24 days while its tip remained in the stomach. On day 24, when diarrhea first began, inadvertent traction by the patient or caregivers probably pulled the tube out of the stomach and into the gastrocolonic channel. Isolated from the peritoneal cavity, the tube never floated free in the peritoneal space during such migration, and therefore the patient never developed peritonitis. Between days 24 and 62, the formula sometimes primarily entered the stomach when it was properly digested (diarrhea-free days), and sometimes the colon bypassing digestion in the small intestine (days with diarrhea). On day 62, the tube fully withdrew into the colon and resulted in the tell-tale signs of CCF: feculent odor from the gastrostoma and undigested formula in the stools. ${ }^{1,2,8}$ The initial 24-day asymptomatic interval should not detract from this hypothesis. Patients with a CCF have remained asymptomatic for up to 2 years, until their tube withdrew to the colon or a replacement tube stayed in the colon without reaching the stomach. ${ }^{5,8}$

An unlikely explanation for this CCF would be that a properly inserted PEG tube later eroded the gastric wall and then gradually penetrated into the adjacent colon. This mechanism has also been described previously, ${ }^{11}$ and would be supported by the initial uneventful 24 days. However, our patient never developed the peritonitis one would have expected while the migrating PEG tip lay free in the peritoneal cavity. It is unlikely that the tube's soft mushroom tip could slowly erode into the colon without first producing a massive free intraperitoneal fluid collection, which was clearly absent on the computed tomography scan (Figure 1).

Several factors caused a delay in the diagnosis of this CCF. First, the patient tolerated the PEG feedings uneventfully for the initial 24 days. Therefore, no one suspected that the delayed-onset diarrhea was related to the PEG. We usually expect a surgical complication to manifest itself during the immediate postoperative period. Second, the patient's symptoms were intermittent. One would expect persistent diarrhea with a CCF. Third, the 
cardinal symptoms indicating a CCF (undigested formula in the feces and feculent odor from the PEG stoma) were not documented till day 62. A foul odor was noted on day 8 but was not investigated further. Fourth, another early diagnostic opportunity was missed on day 8 when the cellulitis at the gastrostoma, which developed after insertion, was treated without a bacterial culture. Potential growth of colonic bacteria on that culture would have suggested fecal etiology of the cellulitis, and hinted at the colonic penetration. Fifth, patient usually wore diapers because of bowel and urinary incontinence. Because caregivers do not routinely check the diaper after each feeding, they probably missed identifying postprandial diarrhea. Also, patient was unable to verbalize and therefore could not report the diarrhea. Most importantly, and probably just because of its rarity, staff did not suspect the CCF, although they did much to rule out other potential causes of diarrhea.

CCFs are indeed rare. Friedmann et $\mathrm{al}^{1}$ observed only 6 CCF cases among 2384 PEGs $(0.25 \%$ incidence). Their PubMed and MEDLINE search identified only 22 other PEG-related CCFs in adults between 2001 and 2005. Ponsky et $\mathrm{a}^{11}$ had one CCF among 227 PEGs (0.4\% incidence). During 30 years, this is our first case of a CCF among approximately 200 PEGs in our residents ( $0.5 \%$ incidence).

This case illustrates that inadvertent colonic penetration can occur even when an experienced gastroenterologist inserts the PEG. To prevent this complication, the colon must be carefully moved out of the way by adequate gastric insufflation with air. Apposition of the gastric wall against the abdominal wall should be verified by finger indentation and transillumination in the epigstrium. ${ }^{1,8}$ At the end of the procedure, the tube's tip should be endoscopically verified to be in the stomach. Premature gastric desufflation should be prevented by applying cricoid pressure during the finder-needle insertion. Some experts even recommend abdominal CAT scans before PEGs are inserted to assess spatial relationship between the stomach and the colon, particularly in patients who have had prior abdominal surgery. ${ }^{8-10}$ However, the latter is not a standard practice.

For suspected PEG misplacements the test of choice is a tubogram, not abdominal radiographs, CAT scan, or colonoscopy. ${ }^{1,2,8}$ Tubograms are easier to perform and can demonstrate the tube's location, antegrade and retrograde filling, gastrocolic or colocutaneous fistulization, and peritoneal leak- age. However, if the PEG tip is partially in the peritoneal cavity, a tubogram would pose a risk of peritonitis from extrusion of the contrast dye. Therefore, only a water-soluble contrast such as gastrograffin should be used.

Clinicians who care for patients with a PEG should suspect a CCF if there is recurrent diarrhea or fecal odor from the gastrostoma, and should confirm the diagnosis by a tubogram. Delayed onset or intermittent occurrence of symptoms does not rule out a CCF.

The authors are grateful to Dilip Patel, MD, for clinical information and Heather Bell for medical records.

\section{References}

1. Gauderer MWL. Percutaneous endoscopic gastrostomy-20 years later: a historical perspective. J Pediatr Surg 2001;36:217-9.

2. Guloglu R, Taviloglu K, Alimoglu O. Colon injury following percutaneous endoscopic gastrostomy tube insertion. J Laparoendosc Adv Surg Tech 2003;13: 69-72.

3. Sherwin P, Schrag S, Sharma S, et al. Complications related to percutaneous endoscopic gastrostomy (PEG) tubes. A comprehensive clinical review. J Gastrointestin Liver Dis 2007;16:407-18.

4. Sasaki T, Fukumori D, Sakai K, Sato M, Ohmori H, Yamamoto F. The safety and feasibility of percutaneous endoscopic gastrostomy placement. Hepatogastroenterology 2004;51:1062-5.

5. Kinoshita Y, Udagawa H, Kajiyama Y, et al. Cologastric fistula and colonic perforation as a complication of percutaneous endoscopic gastrostomy. Surg Laparosc Endosc Percutan Tech 1999;9:220-2.

6. Berger SA, Zarling EJ. Colocutaneous fistula following migration of PEG tube. Gastrointest Endosc 1991;37:86-8.

7. Tong K, Khan Z. Unexplained diarrhea in a patient with a percutaneous endoscopy gastrostomy (PEG) tube. Endoscopy 2007;39(Suppl 1):E69.

8. Friedmann R, Feldman H, Sonnenblick M. Misplacement of percutaneously inserted gastrostomy tube into the colon: report of 6 cases and review of the literature. JPEN J Parenter Enteral Nutr 2007; 31:469-76.

9. Vogt W, Messmann H, Lock G, et al. CT-guided PEG in patients with unsuccessful endoscopic transillumination. Gastrointest Endosc 1996;43:138-40.

10. Roozrokh HC, Ripepi A, Stahlfeld K. Gastrocolocutaneous fistula as a complication of peg tube placement. Surg Endosc 2002;16:538-9.

11. Ponsky J, Gauderer M, Stellato T, Aszodi A. Percutaneous approaches to enteral alimentation. Am J Surg 1985;149:102-5. 